\title{
An Improved Chirplet Transform and Its Application for Harmonics Detection
}

\author{
Guo-Sheng $\mathrm{Hu}^{1}$, Feng-Feng Zhu ${ }^{2}$ \\ ${ }^{1}$ School of Computer, Shanghai Technical Institute of Electronics and Information, Shanghai, China \\ ${ }^{2}$ School of Mathematics Science, South China University of Technology, Guangzhou, China \\ E-mail: jamhu8@sohu.com,Zhuffeng@126.com \\ Received January 2, 2011; revised March 1, 2011; accepted March 8, 2011
}

\begin{abstract}
The chirplet transform is the generalization form of fast Fourier Transform, short-time Fourier transform, and wavelet transform. It has the most flexible time frequency window and successfully used in practices. However, the chirplet transform has not inherent inverse transform, and can not overcome the signal reconstructing problem. In this paper, we proposed the improved chirplet transform (ICT) and constructed the inverse ICT. Finally, by simulating the harmonic voltages, the power of the improved chirplet transform are illustrated for harmonic detection. The contours clearly showed the harmonic occurrence time and harmonic duration.
\end{abstract}

Keywords: Harmonics, Improved Chirplet Transform (ICT), S-Transform, Time-Frequency Representation (TFR)

\section{Introduction}

In many power quality analysis and disciplines, the concept of a stationary time series is a mathematical idealization that is never realized and is not particularly useful in the detection of power quality disturbances in power systems. Although the Fourier transform of the entire time series does contain information about the spectral components in a time series, for a large class of practical applications such as voltage signals in power systems, this information is inadequate. So in the year of 1996, Stockwell, Mansinha and Lowe presented a new S transform that provides a joint time-frequency representation (TFR) with frequency-dependent resolution [1] while, at the same time, maintaining the direct relationship, through time-averaging, with the Fourier spectrum. Several have been proposed in the past; among them are the Gabor transform [2], the related short-time Fourier transforms [3], the continuous wavelet transform (CWT) [4], and the bilinear class of time-frequency distributions known as Cohen's class [5], of which the Wigner distribution [6] is a member. The $\mathrm{S}$ transform was defined as following [1].

$$
S(\tau, f)=\int_{-\infty}^{+\infty} h(t) \frac{|f|}{\sqrt{2 \pi}} \mathrm{e}^{-\frac{(\tau-t)^{2} f^{2}}{2}} \mathrm{e}^{-j 2 \pi f t} \mathrm{~d} t .
$$

It is easy to prove that

$$
\int_{-\infty}^{+\infty} S(\tau, f) \mathrm{d} \tau=H(f) .
$$

where $H(f)$ is the Fourier transform of $h(t)$. It follows that $h(t)$ is exactly recoverable from $S(\tau, f)$. Thus

$$
h(t)=\int_{-\infty}^{+\infty}\left\{\int_{-\infty}^{+\infty} S(\tau, f) \mathrm{d} \tau\right\} \mathrm{e}^{j 2 \pi f t} \mathrm{~d} f .
$$

The S-transforms have successfully been used in power system disturbance detection and identification [7]. Another time-frequency representation, Chirplet transform (CT), was defined by Mann Steven in 1992 [8].

$$
C(b, \omega)=\frac{1}{\sqrt{2 \pi} \sigma} \int_{-\infty}^{+\infty} h(t) \mathrm{e}^{-\frac{(t-b)^{2}}{2 \sigma^{2}}} \mathrm{e}^{-i\left(\omega t+q t^{2}\right)} \mathrm{d} t .
$$

From (4), it is easy to show that Chirplet transform is an extension of Gabor transform, short-time Fourier transform and continuous wavelet transform, furthermore, the time-frequency window of CT is more flexible than that of CWT. CT has been used successfully to classify power quality disturbance (including voltage sag, voltage swell, voltage interruption, and linear time-varying harmonics and nonlinear time-varying harmonics) [9], denoise the motor fault signals [10], and detect slight fault of electrical machines [11]. 
However, CT does not satisfy basic characters (2) and (3) and inconvenience in practical applications. So, in this paper, we present an improved Chirplet transform satisfying (2) and (3), moreover, the novel Chirplet transform is an extension of the $S$-transform.

This paper is organized as follows. The improved Chirplet transform is presented in Section 2, and the numerical algorithm is introduced in Section 3; Several simulated power quality harmonic waveforms are detected and identified using the proposed method in Section 4; Finally, The conclusions and references are given.

\section{The Improved Chirplet Transform}

Chirplet transform is considered as the "phase correction" of the CWT. The CWT $W(b, a)$ of a function $h(t)$ is defined by [4]

$$
W(b, a)=\int_{-\infty}^{+\infty} h(t) w(t-b, a) \mathrm{d} t .
$$

where $w(\bullet)$ is a scaled replica of the fundamental mother wavelet. The dilation $a$ determines the "width" of the wavelet $w(\bullet)$ and thus controls the resolution. Along with (5), there exists an admissibility condition on the mother wavelet $w(\bullet)$ [4] that $w(\bullet)$ must have zero mean.

The improved chirplet transform of a function $h(t)$ is defined as a CWT with a specific mother wavelet defined as

$$
w(t, f)=\frac{\sqrt{f^{2}+i q}}{\sqrt{2 \pi}} \mathrm{e}^{-\frac{t^{2}\left(f^{2}-i q\right)}{2}} \mathrm{e}^{-i\left(2 \pi f t+q t^{2}\right)} .
$$

note that the frequency $f$ and constant $q$.

The improved chirplet wavelet in (6) does not satisfy the condition of zero mean for an admissible wavelet; therefore, it is not strictly a CWT, Written out explicitly, the improved chirplet transform is

$$
\begin{aligned}
& I C(b, f) \\
& =\frac{\sqrt{f^{2}+i q}}{\sqrt{2 \pi}} \cdot \int_{-\infty}^{+\infty} h(t) \mathrm{e}^{-\frac{(t-b)^{2}\left(f^{2}-i q\right)}{2}} \mathrm{e}^{-i\left(2 \pi f+q(t-b)^{2}\right)} \mathrm{d} t .
\end{aligned}
$$

It is obvious that Equation (7) degenerates to be an $S$-transform as $q=0$ [1].

If the improved chirplet transform is indeed a representation of the local spectrum, one would expect a simple operation of averaging the local spectra over time to give the Fourier spectrum. It is shown as follows

$$
\int_{-\infty}^{+\infty} I C(b, f) \mathrm{d} b=H(f) .
$$

where $H(f)$ is the Fourier transform of $h(t)$. It follows that $h(t)$ is exactly recoverable from $\operatorname{IC}(b, f)$. Thus

$$
h(t)=\int_{-\infty}^{+\infty}\left\{\int_{-\infty}^{+\infty} I C(b, f) \mathrm{d} b\right\} \mathrm{e}^{i 2 \pi f t} \mathrm{~d} f .
$$

Proof: from

$$
\begin{aligned}
& \int_{-\infty}^{+\infty} \mathrm{e}^{-\frac{(t-b)^{2}\left(f^{2}-i q\right)}{2}} \mathrm{e}^{-i q(t-b)^{2}} \mathrm{~d} b=\int_{-\infty}^{+\infty} \mathrm{e}^{-\frac{(t-b)^{2}\left(f^{2}+i q\right)}{2}} \mathrm{~d} b \\
& =\frac{1}{\sqrt{f^{2}+i q}} \int_{-\infty}^{+\infty} \mathrm{e}^{-\frac{u^{2}}{2}} \mathrm{~d} u\left(\text { set } u=\sqrt{f^{2}+i q}(b-t)\right) \\
& =\frac{\sqrt{2 \pi}}{\sqrt{f^{2}+i q}}\left(\because \int_{-\infty}^{+\infty} \mathrm{e}^{-\frac{u^{2}}{2}} \mathrm{~d} u=\sqrt{2 \pi}\right) .
\end{aligned}
$$

we can get

$$
\begin{aligned}
& \int_{-\infty}^{+\infty} I C(b, f) \mathrm{d} b \\
& =\int_{-\infty}^{+\infty} \frac{\sqrt{f^{2}+i q}}{\sqrt{2 \pi}} \cdot \int_{-\infty}^{+\infty} h(t) \mathrm{e}^{-\frac{(t-b)^{2}\left(f^{2}-i q\right)}{2}} \mathrm{e}^{-i\left(2 \pi f t+q(t-b)^{2}\right)} \mathrm{d} t \mathrm{~d} b \\
& =\frac{\sqrt{f^{2}+i q}}{\sqrt{2 \pi}} \cdot \int_{-\infty}^{+\infty} h(t) \mathrm{e}^{-i 2 \pi f t} \mathrm{~d} t \int_{-\infty}^{+\infty} \mathrm{e}^{-\frac{(t-b)^{2}\left(f^{2}-i q\right)}{2}} \mathrm{e}^{-i\left(q(t-b)^{2}\right)} \mathrm{d} b . \\
& =\int_{-\infty}^{+\infty} h(t) \mathrm{e}^{-i 2 \pi f t} \mathrm{~d} t=H(f) .
\end{aligned}
$$

Then (9) is the inverse of the above equation.

Alike FFT, STFT, WT, and CT, the improved chirplet transform has the linear property. This is an advantage over the bilinear class of time-frequency representations (TFR's). The presence of the cross terms makes it difficult to reliably estimate the signal. The improved chirplet transform can be written as operations on the Fourier spectrum $H(f)$ of $h(t)$

$$
I C(b, f)=\int_{-\infty}^{+\infty} H(\alpha+f) \mathrm{e}^{-\frac{2 \pi^{2} \alpha^{2}}{f^{2}+i q}} \mathrm{e}^{i 2 \pi \alpha b} \mathrm{~d} \alpha
$$

Proof:

$$
\begin{aligned}
& \int_{-\infty}^{+\infty} H(\alpha+f) \mathrm{e}^{-\frac{2 \pi^{2} \alpha^{2}}{f^{2}+i q}} \mathrm{e}^{i 2 \pi \alpha b} \mathrm{~d} \alpha \\
& =\int_{-\infty}^{+\infty} \int_{-\infty}^{+\infty} h(t) \mathrm{e}^{-i 2 \pi(\alpha+f) t} \mathrm{e}^{-\frac{2 \pi^{2} \alpha^{2}}{f^{2}+i q}} \mathrm{e}^{i 2 \pi \alpha b} \mathrm{~d} t \mathrm{~d} \alpha \\
& =\int_{-\infty}^{+\infty} h(t) \mathrm{e}^{-i 2 \pi f t} \mathrm{e}^{-\left(-\frac{f^{2}+i q}{2}(t-b)^{2}\right)} \mathrm{d} t \int_{-\infty}^{+\infty} \mathrm{e}^{-\left(\frac{\sqrt{2} \pi \alpha}{\sqrt{f^{2}+i q}}\right)^{2}} \mathrm{~d} \alpha \\
& =\frac{\sqrt{f^{2}+i q}}{\sqrt{2 \pi}} \cdot \int_{-\infty}^{+\infty} h(t) \cdot \mathrm{e}^{-\left(-\frac{(t-b)^{2}\left(f^{2}+i q\right)}{2}\right)} \mathrm{e}^{-i\left(2 \pi f t+q(t-b)^{2}\right)} \mathrm{d} t \\
& =I C(b, f) .
\end{aligned}
$$

The discrete analog of (11) is used to compute the discrete improved chirplet transform by taking advantage of the efficiency of the Fast Fourier transform (FFT) and the convolution theorem. 


\section{The Discrete Improved Chirplet Transform}

Let $h[k T], k=0,1,2, \cdots, N-1$ denote a discrete time series corresponding to signal $h(t)$ with a time sampling interval of $T$. The discrete Fourier transform is given by

$$
H\left[\frac{n}{N T}\right]=\frac{1}{N} \sum_{k=0}^{N-1} h[k T] \mathrm{e}^{-\frac{i 2 \pi n k}{N}} .
$$

where $n=0,1,2, \cdots, N-1$. In the discrete case, the improved chirplet transform is the projection of the vector defined by the time series $h[k T]$ onto a spanning set of vectors. The spanning vectors are not orthogonal, and the elements of the improved chirplet transform are not independent. Each basis vector (of the Fourier transform) is divided into $N$ localized vectors by an elementby-element product with the $N$ shifted Gaussians such that the sum of these $N$ localized vectors is the original basis vector.

Using (11), the improved chirplet transform of a discrete time series $h[k T]$ is given by (letting $f \rightarrow n / N T$ and $\tau \rightarrow j T$ )

$$
\operatorname{IC}\left[j T, \frac{n}{N T}\right]=\sum_{m=0}^{N-1} H\left[\frac{m+n}{N T}\right] \mathrm{e}^{-\frac{2 \pi^{2 m^{2}}}{n^{2}+i \tilde{q}}} \mathrm{e}^{\frac{i 2 \pi m j}{N}} .
$$

where $n \neq 0, \tilde{q}=q(N T)^{2}$. For the $n=0$, it is equal to the constant defined as

$$
\operatorname{IC}[j T, 0]=\frac{1}{N} \sum_{m=0}^{N-1} h\left(\frac{m}{N T}\right) .
$$

with $j, m$, and $n=0,1,2, \cdots, N-1$. The discrete improved chirplet transform suffers the familiar problems from sampling and finite length, giving rise to implicit periodicity in the time and frequency domains. The discrete inverse of the improved chirplet transforms (13) and (14) is

$$
h[k T]=\sum_{k=0}^{N-1}\left\{\frac{1}{N} \sum_{j=0}^{N-1} I C\left[j T, \frac{n}{N T}\right]\right\} \mathrm{e}^{\frac{j 2 \pi n k}{N}} .
$$

\section{Examples}

\subsection{The Signal TFR Figure Using the Improved Chirplet Transform}

Equations (7) and (9) are the improved chirplet transform (ICT) for the time-frequency representation (TFR) and its inverse ICT for the signal reconstruction. In the follow figures, Figure 1(a) is the A-phase current signal of the inductor motor with single phase grounding. Figure 2(b) shows the TFR of the A-phase current signal of the motor using (7). The reconstruction signal using (9) is illustrate in Figure 2(c). From Figure 1, we know (7) and (9) are very effective for representing a time frequency feature of a signal and reconstructing from TFR.

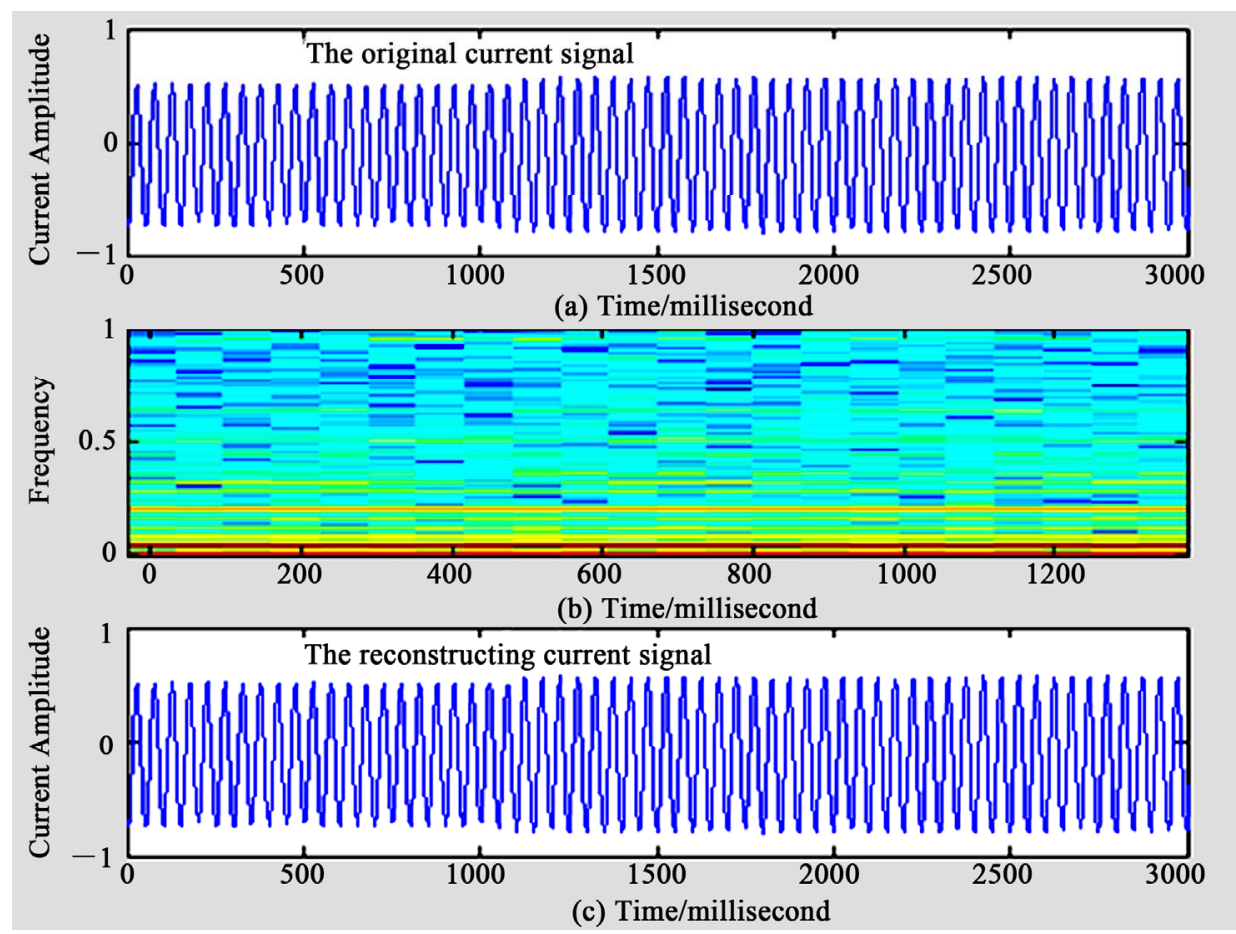

Figure 1. The motor A-phase current signal (a), its TFR (b), and the reconstructing signal. 


\subsection{The harmonics Detection}

Figures 2 and $\mathbf{3}$ demonstrate the class of time series for which the improved chirplet transform would be useful; they highlight the advantages of such an approach as compared with other techniques.

Considering a simulating segment harmonic voltage with zero initial phase as follows.

$$
f(t)=\left\{\begin{array}{l}
\sin (2 * p i * 50 * t) \quad(0 \leq t \leq 0.3) \\
\sin (2 * p i * 50 * t)+\sin (2 * p i * 150 * t) \quad(0.3<t \leq 0.6) \\
\sin (2 * p i *(50+0.2 * t) * t) \quad(0.6<t \leq 1.4) \\
\sin (2 * p i * 50 * t)+\sin (2 * p i * 350 * t) \quad(1.4<t \leq 1.7) \\
\sin (2 * p i * 50 * t) \quad(1.7 \leq t \leq 2)
\end{array}\right.
$$

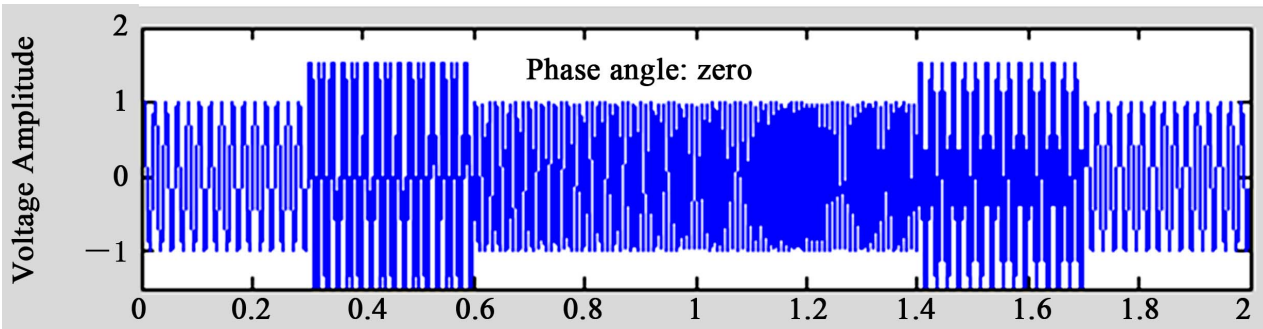

(a) Time/second

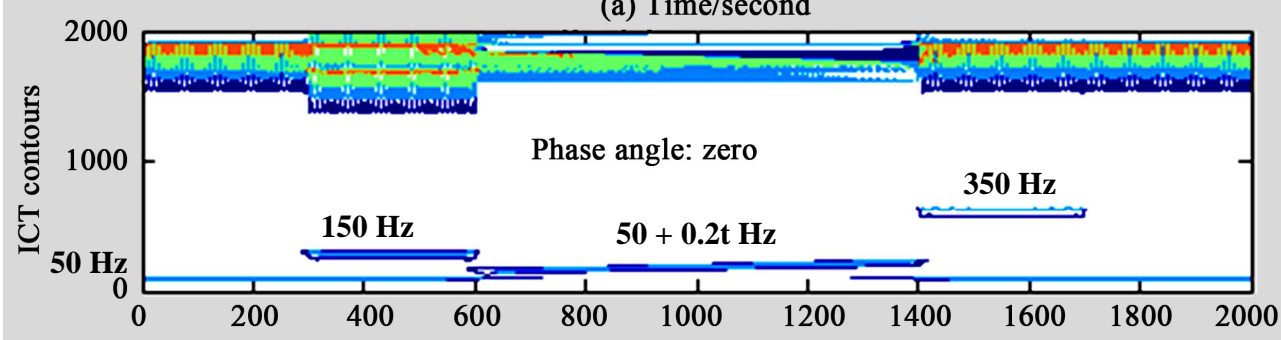

(b) Sampling Points/n

Figure 2. The simulating voltage with zero initial phase (a), and its ICT contour (b).

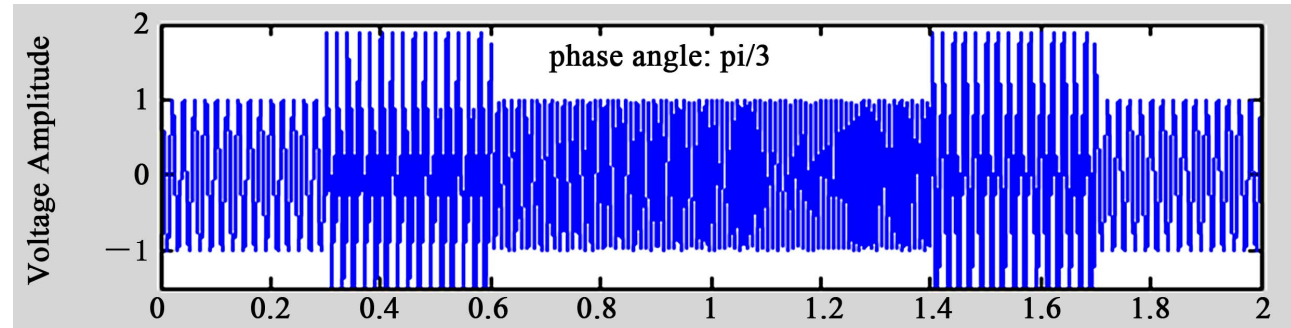

(a) Time/second

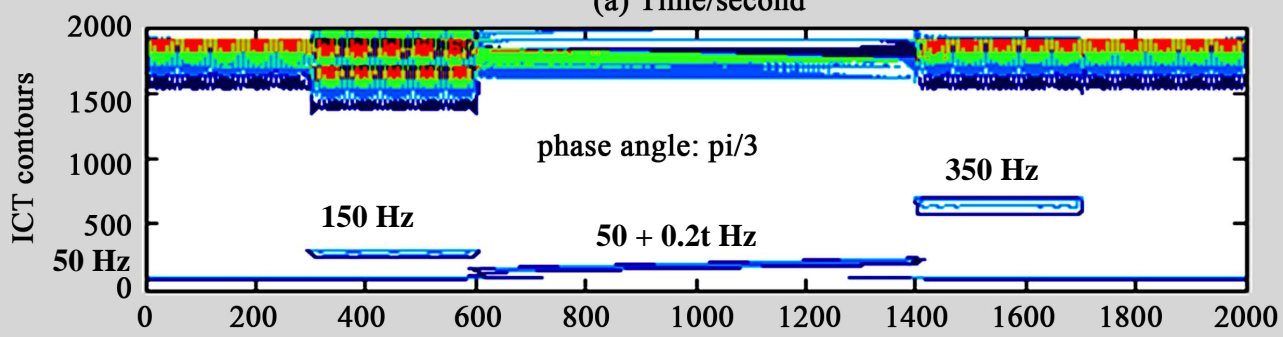

(b) Sampling Points/n

Figure 3. The simulating voltage with pi/3 initial phase (a), and its ICT contour (b). 
The voltage signal divided into 5 time segments. In the first time interval, the voltage constrains only a frequency: $50 \mathrm{~Hz}$. At time 0.3 , the voltage constrains another harmonic: $150 \mathrm{~Hz}$. In the time interval $0.6<t \leq 1.4$, the voltage constrains a linear time changing harmonic. Then at $1.4 \mathrm{~s}$, the harmonic, $350 \mathrm{~Hz}$, added to the signal. Finally, the voltage retain normally at $1.7 \mathrm{~s}$.

The sampling frequency is $1000 \mathrm{~Hz}$. Figure 2 (a) is the improved chirplet transform plot of (16). Figure 2(b) is the contour plot of the signal (a) using (13) and (14). Form Figure 2(b), we find the ICT contour illustrates the work frequency $50 \mathrm{~Hz}$, two harmonic frequency $150 \mathrm{~Hz}$ and $350 \mathrm{~Hz}$, and the linear time changing frequency $50+$ $0.2 \mathrm{t} \mathrm{Hz}$.

Moreover, the contour in Figure 2(b) clearly shows the harmonic occurrence times and durations.

In order to investigate the influence of initial phase, we modulating the above simulating voltage signal with pi/3 phase. From Figure 3, we find that the initial phase does not influence the harmonic detection.

\section{Conclusions}

The chirplet transform is the generalization form of fast Fourier transform, short-time Fourier transform, and wavelet transform. It has the most flexible time frequency window and successfully used in practices. However, the chirplet transform has not inherent reconstructing formulae. So we proposed the improved chirplet transform (ICT) and constructed the inverse ICT. Finally, the power of the improved chirplet transform is apparent from the above examples.

\section{References}

[1] R. G. Stockwell, L. Mansinha and R. P. Lowe, "Location of the Complex Spectrum: The S-Transform," IEEE Transactions on Signal Processing, Vol. 44, No. 4, 1996, pp. 998-1001. doi:10.1109/78.492555

[2] D. Gabor, "Theory of Communication," Journal of Institution of Electrical Engineers, Vol. 93, No. 3, 1946, pp. 429-457.

[3] R. N. Bracewell, "The Fourier Transform and Its Applications," McGraw-Hill, New York, 1978.

[4] S. Mallat, "A Wavelet Tour of Signal Processing," 2nd Edition, Academic Press, Waltham, 2001.

[5] L. Cohen, "Time-Frequency Distributions-A Review," Proceedings of the IEEE, Vol. 77, No. 7, 1989, pp. 941-981. doi:10.1109/5.30749

[6] F. Hlawatsch and G. F. Boudreuax-Bartels, "Linear and Quadratic Time-Frequency Signal Representations," Proceedings of Signal Processing Magazine, Vol. 9, No. 2, 1992, pp. 21-67.

[7] M. V. Chilukur and P. K. Dash, "Multiresolution S-Transform-Based Fuzzy Recognition System for Power Quality Events," IEEE Transactions on Power Delivery, Vol. 19, No. 1, 2004, pp. 323-330. doi:10.1109/TPWRD.2003.820180

[8] S. Mann and S. Haykin, "The Chirplet Transform: Physical Considerations," IEEE Transactions on Signal Processing, 1995, Vol. 43, No. 11, pp. 2745-2761. doi: $10.1109 / 78.482123$

[9] G.-S. Hu, F.-F. Zhu and Y.-J. Tu, "Power Quality Disturbance Detection and Classification Using Chirplet Transform," Lecture Notes in Computer Science, Vol. 4247, 2006, pp. 34-41. doi:10.1007/11903697_5

[10] Z. Ren, G. S. Hu, W. Y. Huang and F. F. Zhu, "Motor Fault Signals Denosing Based on Chirplet Transform," Transactions of China Electrotechnical Society, Vol. 17, No. 3, 2002, pp. 59-62.

[11] G. S. Hu and F. F. Zhu, "Location of slight Fault in Electric Machine Using Trigonometric Spline Chirplet Transforms," Power System Technology, Vol. 27, No. 2, 2003, pp. 28-31. 